$\begin{array}{ll}\text { le portiQue } & \text { Le Portique } \\ \text { Revue de philosophie et de sciences humaines }\end{array}$

$20 \mid 2007$

Gilles Deleuze et Félix Guattari : Territoires et devenirs

\title{
Devenirs, devenir écrivain, Proust-Kafka
}

\section{Philippe Mengue}

\section{OpenEdition}

\section{Journals}

Édition électronique

URL : http://journals.openedition.org/leportique/1369

DOI : $10.4000 /$ leportique.1369

ISSN : $1777-5280$

\section{Éditeur}

Association "Les Amis du Portique"

Édition imprimée

Date de publication : 15 décembre 2007

ISSN : 1283-8594

\section{Référence électronique}

Philippe Mengue, «Devenirs, devenir écrivain, Proust-Kafka », Le Portique [En ligne], 20 | 2007, mis en ligne le 06 novembre 2009, consulté le 25 mars 2021. URL : http://journals.openedition.org/leportique/ 1369 ; DOI : https://doi.org/10.4000/leportique.1369

Ce document a été généré automatiquement le 25 mars 2021.

Tous droits réservés 


\title{
Devenirs, devenir écrivain, Proust- Kafka
}

\author{
Philippe Mengue
}

1 Je voudrais, dans le cadre de notre colloque, "Territoires et devenirs ", et pour mieux problématiser le concept de devenir, questionner avec vous ce qu'il en est précisément du «devenir écrivain » selon Gilles Deleuze. Il m'a semblé pertinent de rattacher ce problème au cas de Proust (étudié, comme on sait par Deleuze dans son remarquable Proust et les signes) qui me servira de point de départ. Je m'aiderai de Kafka, à travers l'analyse que Gilles Deleuze et Félix Guattari en ont donné en 1975 dans Kafka, Pour une littérature mineure ${ }^{1}$. D'où le titre de cette intervention qui associe Proust et Kafka autour de la question du devenir ${ }^{2}$.

\section{Devenir écrivain}

On peut deviner tout de suite de quoi il retourne.

3 Il arrive en effet à Deleuze contre toute attente, et en prenant à rebours le bon sens critique des historiens de la littérature, de déclarer que toute "grande " littérature (soit, à ses yeux toute littérature mineure seule réellement créatrice) s'occupe de devenirs au pluriel et surtout pas d'un prétendu devenir écrivain :

Écrire est un devenir, écrire est traversé d'étranges devenirs qui ne sont pas des devenirs-écrivains » (Mille plateaux, p. 293). «Écrire, c'est devenir, mais ce n'est pas du tout devenir écrivain. C'est devenir autre chose (Dialogue, p. 54).

Évidemment, on songe tout de suite à Proust qui rend cette déclaration paradoxale, intenable même, à première vue. Ou bien Proust n'écrit pas, n'est pas un grand écrivain (ce qu'on ne peut admettre, et qui va contre le jugement explicite de Deleuze), ou bien il est à tenir pour tel, et la sentence de Deleuze reçoit apparemment une réfutation empirique qui l'invalide complètement. En effet, pour qui a lu la Recherche, ne serait-ce qu'en partie, de quoi d'autre s'agit-il donc que du récit du devenit-écrivain du narrateur? Quelle intrigue, quelle action trouve-t-on dans la Recherche? Pratiquement aucune, hormis le douloureux apprentissage de Marcel, et son devenir écrivain. Que 
nous raconte d'essentiellement autre cette œuvre que sa désolation, son regret de ne pouvoir écrire, de ne pas avoir de dons littéraire, de son désir d'être artiste et comment, grâce à tout un long parcours, qui est celui de sa vie, il est conduit à la découverte, «la durée d'un éclair », « d'un peu de temps à l'état pur » (R, III, 872), soit l'expérience d'une forme d'éternité "hors temps" qui seule fonde sa résolution d'écrire et de devenir écrivain?

Proust, à la fin de la Recherche - et dans le sillage de l'illumination éprouvé lors de sa visite à l'hôtel de Guermantes, expérience extatique de la « résurrection » (R, III, 874) du passé dans sa splendeur, "joie du réel retrouvé » (R, III, 879) et tel qu'il ne fut jamais vécu - écrit lui-même : «Ainsi toute ma vie jusqu'à ce jour aurait pu et n'aurait pas pu être résumée sous ce titre : Une vocation. Elle ne l'aurait pas pu en ce sens que la littérature n'avait joué aucun rôle dans ma vie. Elle l'aurait pu en ce que cette vie, les souvenirs de ses tristesses, de ses joies, formaient une réserve ... » (R, III, 899) qui a servi de matière à l'écrivain ${ }^{3}$ qu'il devient, est déjà devenu.

5 Mon choix de Proust s'éclaire donc: il semble faire directement objection, et de manière irrécusable, aux déclarations péremptoires, prises à la lettre de Gilles Deleuze. Qu'en est-il ? Mon objectif n'est pas de contredire ou réfuter Deleuze. Ce serait ridicule. L'« explication » de Deleuze et avec Deleuze me permettra de dégager un problème plus fondamental qui engage non seulement la théorie des devenirs mais le sens même de la littérature.

\section{L'écriture et la vie}

6 À première vue, et pour le fond, d'une façon générale qui déborde Proust, on ne voit pas comment il serait possible de dissocier les devenirs dont la littérature seraient porteuse ou créatrice, du devenir écrivain dans lequel se trouve en apparence nécessairement pris celui qui écrit, puisque c'est lui qui invente ou a expérimenté les devenirs qu'il nous rapporte et qu'en nous les rapportant il devient par là même écrivain.

7 À cet argument général de bon sens, s'en ajoute un autre, qui met en jeu une exigence propre à la pensée de Gilles Deleuze. Pour lui, en effet, il ne peut être question de dissocier ou opposer la vie et l'œuvre ${ }^{4}$. La littérature ne cesse de répéter Deleuze, avec les auteurs anglo-américains qu'il affectionne tant, a pour fonction la libération de la vie partout où elle se trouve emprisonnée. Qu'on se reporte au mot célèbre mot d'ordre nietzschéen et spinosiste :

Porter la vie à la puissance absolue ou de "grande Santé ». [...] L'écriture n'a pas sa

fin en soi-même. [...] L'écriture a pour seule fin la vie ... (Dialogue, p. 12). L'art n'est jamais une fin, il n'est qu'un instrument pour tracer des lignes de vie, c'est-à-dire tous ces devenirs réels qui ne se produisent pas seulement dans l'art (Mille plateaux, p. 239).

8 Si donc on demande avec Deleuze : «Pourquoi écrit-on?» (Dialogue, p. 61), on voit d'abord, comme il le dit, "qu'il ne s'agit pas d'écriture " (p.61) - au sens où les textualistes par exemple ont pris ce terme - mais qu'il s'agit de la vie. Mais qu'entendre par vie ? Deleuze place sa philosophie sous l'idée générale du « vitalisme » (voir « tout ce que j'ai écrit était vitaliste, du moins je l'espère », Pourparlers, p. 196). Ce que Deleuze appelle " vie », n'est ni de l'ordre de la vie des organes, ni de l'existence (existentialisme), ni de l'être-au-monde (heideggérien). On mesure l'écart avec ces concepts quand on réalise que ce qui caractérise la vie dans ce qu'elle a d'intrinsèque, c'est la déterritorialisation. 
La vie dans ce qu'elle a de vitalement créateur, doit être pensée à l'image d'une «ligne abstraite ${ }^{5}$ qui tire les choses et les êtres, les lance dans un devenir autre, les fait littéralement fuir, partir, glisser. Deleuze lui associe souvent les traits d'une ligne de sorcière, d'un souffle, d'un vent, de rafale dans le dos... Un tel processus est celui de la déterritorialisation.

On a donc, en gros (mais cette approximation nous suffira pour le moment) la liste d'équation suivante :

Écrire, c'est devenir ; devenir, c'est déterritorialiser ; déterritorialiser, c'est tracer une ligne abstraite, c'est tracer des lignes de fuite ${ }^{6}$.

9 On connaît quels sont ces devenirs ou ligne de fuite. C'est par rapport à la forme « homme» (forme molaire et majoritaire en ce qu'elle sert de norme) dont ils sont la « minoration » (d'où le terme de littérature mineure) qu'ils sont définissables : devenir enfant, femme, animal, imperceptible (voir surtout Mille plateaux, Plateau 10). De ces devenirs, on en trouvera en effet, dans Proust, et en particulier le gigantesque et effrayant devenir-araignée du narrateur lui-même tel qu'il est génialement décrit dans le dernier chapitre rajouté de Proust et les signes, en 1975. On peut multiplier à loisir tous ces devenirs, le problème n'en subsiste pas moins. Car à travers tous ces devenirs moléculaires, rien n'empêche d'affirmer, comme le fait Proust lui-même, du moins son narrateur, qu'il fait l'apprentissage de l'unique devenir qui le sauve, soit le devenirécrivain, présenté comme son propre salut du, son accès à la vie supérieure, à la grande santé, qui est celle de la littérature.

\section{Devenir et histoire}

Les conditions de possibilité d'une solution au problème de la relégation du devenir écrivain de l'ensemble des devenirs véritables, sont au nombre de deux et, en renversant l'imaginaire habituel que l'on projette sur la littérature, elles posent les fondements de la révolution deleuzienne dans le domaine de la critique littéraire.

$111^{\circ}$ La première opération consiste à défaire l'évidence que nous vivons dans un temps unique et linéaire. À cette vision simpliste Deleuze oppose la conception, issue lointainement du stoïcisme, qui implique la coupure entre deux temps, Aion et Chronos, et qui fonde sa distinction entre l'histoire et le devenir. L'histoire concerne le présent actuel ou l'ensemble de ces présents, comme passé ou l'ensemble de ces présents comme avenir. Mais l'être ou le réel ne se réduit pas à ses actualités, aux états de chose. Il implique une autre dimension, tout aussi réelle, mais virtuelle, et qui, comme devenir virtuel, n'est présent en aucun présent effectif. Les virtualités surplombent ou enveloppent les états de chose actuels, comme événement trop grand pour eux, puisqu'ils sont à la fois toujours déjà passé et toujours prêt à arriver.

L'histoire n'est pas l'expérimentation, elle est seulement l'ensemble des conditions presque négatives qui rendent possible l'expérimentation de quelque chose qui échappe à l'histoire. Sans l'histoire, l'expérimentation resterait indéterminée, inconditionnée, mais l'expérimentation n'est pas historique, elle est philosophique ${ }^{7}$.

Dans ce cadre conceptuel, on comprend que « devenir écrivain " puisse appartenir à l'histoire mais qu'il ne concerne pas les véritables devenirs.

Écrire est une affaire de devenir [...] qui déborde toute matière vivable ou vécue

$(\mathrm{Cr} \& \mathrm{Cl}$, p. 11), soit donc ce qui appartient à l'histoire individuelle ou collective. 
13 Le sujet, ou le contenu, de la Recherche ne peut donc pas consister dans cette autobiographie par laquelle Proust nous narrerait comment il fit la découverte de sa vocation, mais les devenirs dont il a été traversé (devenir femme ou fleur, devenir homosexuel, devenir araignée, etc.).

\section{La fonction auteur}

$142^{\circ}$ La seconde opération qui rend possible la mise à l'écart du faux devenir écrivain consiste à défaire l'évidence qui veut que l'œuvre soit le produit (invention, création...) d'un Auteur. Précédemment on a assisté à l'éviction du contenu, maintenant on assiste à l'éviction de la cause ou de l'origine. Il ne s'agit plus du sujet au sens du thème, du contenu ou objet de la narration (qu'est-ce qui est raconté ?) mais du sujet au sens de celui qui écrit (qui raconte ?). L'auteur n'est ni le narré de prédilection, ni le narrant ou écrivant. Le devenir-écrivain, des deux côtés, objet et sujet, n'a donc plus rien affaire avec la littérature. Mais comment admettre ce dernier postulat concernant l'absence de l'Auteur? Deleuze ne serait donc pas l'auteur de Logique du sens?

La contre-évidence factuelle se dissipe si l'on veut bien prendre en considération que ce qui est évincé c'est,

16 a) d'une part, le sujet souverain, avec une majuscule, au sens philosophique et cartésien, posé comme fondement, centre et origine de ces actes, dont tous ses énoncés dépendent en tant qu'il est posé comme fondamentalement maître des significations et du sens de l'œuvre.

17 C'est aussi, b) d'autre part, une illusion romantique solidaire de l'illusion métaphysique précédente, celle qui veut que l'œuvre, exprime avant tout, comme un effet sa cause, la personnalité de son auteur, son moi, son ego, son génie.

On connaît cette critique, où interfèrent les deux motifs, elle vient de loin, et n'est pas propre à Deleuze. Quand il déclare que la littérature n'est pas une affaire privée, personnelle, qu'

Écrire n'est pas raconter ses souvenirs, ses voyages, ses amours et ses deuils, ses

rêves et ses fantasmes ( $\mathrm{Cr} \& \mathrm{Cl}$, p. 12),

il a parfaitement raison, et ne fait que rejoindre principalement Nietzsche et Valery, et bien évidemment le structuralisme depuis Lévi-Strauss.

Valéry déjà, dans Tel quel, avait congédié la fonction auteur quand il déclarait : « toute œuvre est l'œuvre de bien d'autres choses qu'un auteur ${ }^{8}$. "Il ne faut jamais conclure de l'œuvre à un homme - mais de l'œuvre à un masque -, et du masque à la machine » 9 .

Ce passage de Valery est clair et tout à fait pré-deleuzien. À la place du sujet souverain on a la machine littéraire, soit une instance non personnelle, non intentionnelle, dans laquelle l'auteur n'est qu'un nom qui occupe la place d'un sujet assujetti, la fonction d'un agent, d'un support au sein d'une machinerie dont il est seulement une des pièces. Si donc la littérature doit être débarrassée de la fonction Auteur, c'est parce que les fonctions créatrices, productrices ne passent plus par elle, même si cette fonction ne cesse de renaître au plan de l'imaginaire que les gens entretiennent à l'égard de la littérature. À partir de là, on comprend le mépris de Valéry pour la biographie et pour l'histoire littéraire entreprise dans une perspective biographique. Il pouvait aussi 
fustiger le «besoin d'écrire » de ces gens qui encombrent la littérature ${ }^{10}$, de leur moi, de leur prétendue ineffable et chère personne.

Deleuze, prend le relais de cette critique, adossée aux mêmes bases de la déconstruction du sujet. Il le fait avec une ironie mordante dans les premières pages de Critique et Clinique, ainsi que dans le Chapitre II de Dialogue, en pointant que ce désir d'écrire est surtout celui de se raconter, de se mettre en avant, d'étaler son moi, de satisfaire son narcissisme en montrant l'intéressante image de soi qui est la sienne et qu'on offre à l'admiration du lecteur. La généralisation de cette attitude dénature la littérature en lui donnant la forme d'un immense journalisme, d'un universel reportage.

Quel est l'apport théorique propre de Deleuze à ce thème déjà commun à plusieurs auteurs et écoles de pensée? Il réside dans la construction de l'idée d'agencement. L'auteur n'est pas absent, mais en tant qu'agent singulier, Proust, Kafka, il fait partie de ce qu'il appelle, principalement à partir de l'étude sur Kafka, un agencement.

Il n'y a pas de sujet mais des agencements collectifs d'énonciation (Dialogue, p. 36).

\section{nécessairement collective puisqu'elle actualise au sens fort du terme le nouvel} agencement, les forces sociales virtuelles qui frappent à la porte. Actualiser n'est pas représenter dans l'imagination ou la fiction quelque chose de préexistant. L'œuvre de Kafka ne se contente pas de représenter des forces données dans le champ social, car ces forces, tout en étant réelles, sont virtuelles. Les actualiser c'est à la fois les diagnostiquer, les connecter, les amplifier dans un agencement littéraire qui les fait proprement exister. Et que l'agencement soit littéraire et dégage des devenirs virtuels n'empêche pas l'œuvre d'être agissante, d'être immédiatement politique et révolutionnaire, puisque le virtuel qu'elle exprime et actualise dans le texte, est luimême réel et actif. Le scripteur, Kafka, n'est donc plus qu'une pièce de la machine littéraire. Son nom propre ne renvoie plus à aucun individu ou sujet privé d'énonciation, mais seulement à une fonction impersonnelle, la « fonction $K$ » pris dans un champ collectif de variations intensives qu'elle exprime.

La lettre $\mathrm{K}$ ne désigne plus un narrateur ni un personnage, mais un agencement d'autant plus machinique, un agent d'autant plus collectif qu'un individu s'y trouve branché dans sa solitude (Kafka, p. 33).

Gilles Deleuze considère donc que le fait même que la littérature soit un agencement collectif (ainsi que nous l'avons défini et qui concerne la littérature mineure) suffit à la rendre véritablement créatrice et proprement littéraire.

L'énonciation la plus individuelle est un cas particulier d'énonciation collective. C'est même une définition : un énoncé est littéraire lorsqu'il est " assumé » par un Célibataire [ Deleuze donne ce nom à toute singularité artiste] qui devance les conditions collectives de l'énonciation [...] le célibataire actuel et la communauté virtuelle - tous les deux réels - sont les pièces d'un agencement collectif (n. s. Kafka, p. 150). 
30

Dans le procès, ou processus, qui donne à la littérature sa possibilité réelle, il n'est donc de place pour un sujet quelconque («il n'y a pas de sujet », Kafka, p. 33), puisque tous les énoncés sont les rouages de l'agencement collectif d'énonciation qui exprime les puissances diabolique à venir (Kafka, p. 33) ainsi que la communauté nationale qui manque et qui est à venir (Kafka, p. 149). On retrouve un des leitmotiv de Deleuze : à la fois le peuple manque et on n'écrit que pour ce peuple qui manque et qui est à venir.

Voilà, pour l'essentiel, un bloc de concepts deleuzien. C'est le plus voyant, le plus connu. Mais, il y a d'autres fils qui courent dans l'œuvre de Deleuze, lignes plus souples et discrètes que je voudrai maintenant renouer. C'est qu'à mes yeux, un problème reste en suspens. L'auteur, dans sa singularité, peut-il être considéré comme indissociable des agencements collectifs? N'y a-t-il pas des conditions subjectives d'écriture qui sans réintroduire une "personne » et des affaires privées, dote le scripteur d'un minimum d'indépendance vis-à-vis des agencements collectifs? Et n'est-on pas alors obligé de reconnaître l'existence de quelque chose comme un sujet, une subjectivité, une strate subjective différente tant de la personne egotique que de l'agencement collectif d'énonciation où il est censé se fondre? Je vais montrer qu'il y a la possibilité de trouver un véritable devenir écrivain dans l'œuvre de Deleuze elle-même, en respectant les principes fondamentaux de son système?

\section{Le sujet de l'écriture}

On ne peut, je crois, éviter que l'agencement littéraire, la machine littéraire, implique un autre sujet, celui qui sans être le sujet molaire écrivain, est le sujet véritable de l'écriture. En effet, il faut bien que l'agent singulier, le «célibataire », le sujet Kafka, Franz de son prénom, ait été pris dans un devenir littéraire pour qu'il puisse assumer ce rôle d'auteur, même entendu comme la fonction " $K$ », au sein de l'agencement collectif. Il y a donc un autre « devenir littéraire » que celui mis en avant par Deleuze pour le récuser, une autre forme de subjectivation, celle qui est requise en tant que suppôt de l'écriture.

Ce sujet se caractérise par deux traits inséparables.

$1^{\circ}$ Tout d'abord, ce sujet, capable d'écrire, c'est celui qui a perdu le pouvoir de dire Je, celui qui est dessaisi de son moi. On comprend la nécessité de cette dépersonnalisation, déperdition, puisque écrire n'est en rien raconter ses souvenirs. Le sujet de l'écriture doit donc considérer la mémoire comme son ennemi ${ }^{11}$, et donc aussi son moi, formé par toute cette mémoire et ces souvenirs dont son identité est inséparable. Le Moi, infatué de lui-même, comment pourrait-il être ouvert aux intensités qui le traversent en même temps que les variations qui font fuir le champ social? Ce moi, qui fait obstacle aux devenirs, devenu trop encombrant, n'empêche nullement l'existence d'un sujet épuré, sujet au delà du moi, de l'ego, comme pure puissance d'ouverture aux affects, aux intensités. Ce sujet capable de "supporter " cette terrible expérience à laquelle la plus part se dérobent le plus souvent, de la perte du moi, est, par cette épreuve même, un véritable sujet, un "hypokeimenon ", soit un sujet d'expérience ou d'expérimentation. Avant d'être dessaisi par la bureaucratie, qu'il exprimera dans son style sec et sobre, neutre et impersonnel, non sans humour, Franz Kafka a été dessaisi par cette mort du moi, de sa personnalité «propre ». 
De ce sujet désenfatué, désenglué de ses territorialités, de ses territoires locaux et familiaux, religieux et ethniques, rendu à lui-même et à son désir, qui est le véritable sujet de la pensée et de l'écriture, Deleuze, semble-t-il, s'en approche par moments. C'est par exemple le cas quand il analyse le rôle du nom propre, qui est le nom de Personne en particulier.

Le nom propre ne désigne pas un individu: c'est au contraire quand l'individu s'ouvre aux multiplicités qui le traversent de part en part, à l'issue du plus sévère exercice de dépersonnalisation, qu'il acquiert son véritable nom propre " (Mille plateaux, p. 51). Dans Critique et clinique, il précise ce lien, en le connectant au rôle de l'impersonnel dans l'écriture, tel que Maurice Blanchot l'avait dégagé : «Ce ne sont pas les deux premières personnes qui servent de condition à l'énonciation littéraire; la littérature ne commence que lorsque naît en nous une troisième personne qui nous dessaisit du pouvoir de dire Je (le "neutre" de Blanchot) » ( $C R \& C l$, p. 13).

La condition de possibilité de l'écriture ne serait donc pas seulement accrochée à l'agencement collectif d'énonciation, puisque sous lui, ou en lui, se dessine un sujet, ou une subjectivité, qui ne devient pas écrivain sans se constituer dans une rencontre où se produit le devenir lié au vacillement du moi.

\section{Le réel et l'impossible dans la langue}

$2^{\circ}$ En second lieu ce sujet de l'écriture, distinct de l'image de l'écrivain molaire, non seulement est un sujet au moi défait, mais c'est un sujet confronté à l'impossible ou au " dehors ». La rencontre avec le dehors, comme impossible, est la seconde condition qui fait de telle personne singulière un sujet d'écriture. Il y a une incidence du réel, comme absence de sens, sur le pur sujet d'écriture. Avec la perte du sujet souverain, est aussi perdu le sens, le sens de l'existence, de la vie, du monde, dont ce sujet se pense être en droit le maître. Logique $d u$ sens, avait développé la logique de ce sens, en tant qu'irréductible à la signification comme à la désignation, et en tant qu'absent. Il y a donc quelque chose qui vient pointer cette absence (l'objet paradoxal, l'objet $=\mathrm{x}$, dans Logique $d u$ sens) et que j'appellerai pour faire vite le point littéraire, en tant qu'il représente la seconde condition nécessairement expérimenté par le sujet d'écriture dans son devenir littéraire.

Ce qui m'intéresse, c'est que Deleuze, dans ses écrits récents où se déploie la théorie des agencements collectifs d'énonciation, ne renie pas tout à fait cette approche par trop lacanienne telle qu'on peut la retrouver dans l'inspiration qui soutenait Logique du sens. Dans son étude en Préface (de 1970) au livre de Louis Wolfson, le Schizo et les Langues, Deleuze s'approche de ce point littéraire y compris dans son remaniement de 1993 dans Critique et clinique, texte auquel je vais uniquement me référer. Wolfson, à la différence d'Artaud, ne peut être considéré, selon Deleuze, comme un écrivain. Il s'en approche seulement. Qu'est-ce qui l'en empêche? Après avoir posé que l'étudiant schizophrénique «est malade du réel » $(c \& c l$, p. 30), il montre comment ce «réel» comme "dehors» de la vie, à la limite du langage, le drame de Wolfson est de ne pouvoir y avoir accès. L'étudiant en langue met en place dans tout son écrit un procédé linguistique, qui témoigne de la psychose, et qui pousse le langage à sa limite, c'est-àdire qui le déterritorialise. Mais, de ce procédé, Wolfson reste prisonnier car il ne peut accéder à l'envers de cette limite, en recueillir les effets positifs sous formes de figures esthétiques, comme c'est le cas, par exemple, pour Artaud ou Raymond Roussel 
auxquels il est comparé dans le texte de Deleuze. Il ne peut franchir la limite à laquelle l'a conduit son procédé, et dans ce dehors du langage (auquel seul le langage donne accès) affronter les figures d'une vie inconnue. Son procédé reste donc improductif (Ibid., p. 33).

Wolfson reste sur le bord, prisonnier de la folie [...] sans pouvoir arracher à son procédé les figures qu'il ne fait qu'entrevoir à peine ( $\mathrm{C} \& \mathrm{cl}, \mathrm{p} .32)$.

Si donc nous tirons la leçon de cette analyse de la psychose, il semble que celui qui se rend capable d'écrire, est celui qui franchissant la limite s'affronte au réel, au dehors comme impossible, et dont il revient, comme le dit souvent Deleuze, "les yeux rouges ». Ce dehors ne peut être ni désigné ou signifié par les mots ; le langage affronte, de l'autre côté de sa limite, le réel comme inassignable, insensé; il forme une « histoire » dit Deleuze, qui est

plutôt ce qu'il y a d'« impossible » dans le langage, et lui appartient d'autant plus étroitement : son dehors (Ibid., p. 32, souligné dans le texte).

Cette confrontation avec l'impossible, et auquel seul le langage ouvre l'accès, avant de donner matière à des « Visions et des Auditions » (Cr\&cl., p. 16), à de «nouvelles figures de la vie» (p. 33) que l'art a pour but de capter dans les intensités a-formelles et asignifiantes et dans le fourmillement de la vie anorganique, constitue bien la seconde condition d'engendrement du sujet de l'écriture.

41 L'écriture est donc commandée par le réel comme ce qui «ne cesse pas de ne pas s'écrire » (Lacan). Elle est réponse au trou du sens, comme trauma, et c'est par là qu'elle est créatrice d'Idées, de Figures esthétiques, qu'elle est déterritorialisante. Avec Deleuze, à côté de la perte du pouvoir de dire Je, explicitement assumé, l'incidence du réel, comme trou du sens, l'abîme dans le chaos, pointe encore dans le devenir écrivain véritable et n'est pas totalement occulté, même dans les derniers écrits.

Maintenant, il me reste à montrer que le devenir écrivain qui est au centre de la Recherche constitue un véritable devenir au sens deleuzien, et tel que je viens de le dégager.

\section{Proust et le devenir imperceptible de l'écrivain}

43 Il serait trop facile d'opposer l'idéalisme proustien à tendance platonicienne au matérialisme deleuzien des intensités. Ce serait en rester à une vue bien superficielle. Car tous deux se font écho, entrent en résonance depuis leurs séries a-parallèles.

$1^{\circ}$ ) Tout d'abord, il est vrai, Deleuze ne parle pas d'essence en soi à la manière de Proust. Mais, deux concepts nous donne un équivalent deleuzien de l'essence proustienne.

D'une part, Deleuze affirme que l'art n'est pas le chaos, et ne peut l'être. Il en est la " composition" (QQPh, p. 192). L'œuvre détient une consistance qui la fait «tenir toute seule» (QQPh, p. 155), et, comme chez Proust, on trouve l'affirmation d'une stabilité, d'une réalité autonome, échappant à la fluidité du chaos, et à la dissémination des flux, qui lui permet de résister au temps, de se conserver intacte, elle-même, dans son identité propre et singulière, comme le ferait une essence.

L'art conserve, et c'est la seule chose au monde qui se conserve (QQPh, p. 154). 
D'autre part, et en conséquence, Deleuze affirme que l'œuvre "existe en soi » ( $Q Q P h$, p. 155), ce qui veut dire qu'elle échappe au temps, en partie ou relativement,

qu'elle «se conserve en soi, dans l'éternité qui coexiste avec cette courte durée " (QQPh, p. 156). L'œuvre d'art durera éternellement, «en soi », du moins tant que durera son support, toile ou pierre, etc. « Le jeune homme sourira sur la toile autant que celle-ci durera » (QQPh, p. 154).

Il n'en va pas différemment de l'essence proustienne, du moins en tant que fixée dans une œuvre d'art. Deleuze reconnaît à sa manière une forme d'extra-temporalité qui se conserve, et Proust, même s'il en rajoute sur la vision des essences et la promesse d'éternité, ne peut que concéder que cet équivalent deleuzien d'éternité dans le temps est le minimum ontologique à préserver, au delà duquel toute forme d'art ne peut que disparaître.

$2^{\circ}$ ) En second lieu, Proust lui aussi s'y connaissait en devenir imperceptible, malgré ses mondanités, et sa fréquentation des salons parisiens du Faubourg Saint-Germain. C'est qu'il devenait de plus en plus comme une immense araignée ${ }^{12}$, à la fois imperceptible au monde et à lui-même, tapi dans le noir de sa chambre, ne sortant plus, vivant la nuit, couché dans son lit et écrivant ses "paperolles", comme autant de mouches qu'il aurait été capté dans le chaos sans fond et qu'il aurait ramené dans les filets de sa toile, de son réseau d'écriture et qui finiront par faire la Recherche du temps perdu, avec toute la folie, toute la schizophrénie qu'elle contient et qui la conduit souterrainement. C'est au terme de son long exercice de dépersonnalisation, qui métamorphose le narrateur en un " corps-toile-araignée ", que se tient dans son imperceptibilité le devenir écrivain, au dire même de Deleuze :

Le narrateur araignée dont la toile même est la Recherche en train de se faire, de se tisser avec chaque fil remué par tel ou tel signe : la toile et l'araignée, la toile et le corps sont une seule et même machine (Proust et les signes, p. 218).

Quant à la seconde condition, à côté de la dépersonnalisation (et comme devenir insecte dans le cas de Proust), que nous avons relevée comme partie intégrante du devenir écrivain, soit la rencontre avec le non-sens et la réponse qui lui est donnée, c'est bien ce que ne cesse de nous révéler la Recherche, de bout en bout. Le point de nonsens, comme point littéraire et dont la rencontre enclenche le devenir écrivain, a pour Proust un lien avec la béance irréductible du temps. L'essence, Combray en soi, le temps dit retrouvé dans une extase qui procure joie et désir d'écrire, n'annule pas l'expérience du temps comme gouffre, abîme où tout s'engloutit. Tout au contraire. L'œuvre d'art comme capture des forces intensives et a-signifiantes, pour rester œuvre d'art doit traîner avec elle un souffle, un vent, une odeur qui lui vient de la béance ou de l'abîme qu'elle affronte. Une trace du Chaos de l'infini est gardée jusque dans l'Essence extra-temporelle.

50 Je ne donnerai que deux passages de la Recherche, parmi les plus beaux, et sans équivoque, qui témoignent de cette entente du sans-fond et qui fait que le temps retrouvé ne l'est jamais que sur un fond de perte, sur un fond de béance.

51 Le souvenir, la réminiscence involontaire, remonte des Enfers qui forment le fond de nuit et d'oubli de Mnémosyne :

je sens tressaillir en moi quelque chose qui se déplace, voudrait s'élever, quelque chose qu'on aurait désancré, à une grande profondeur ; je ne sais ce que c'est, mais cela monte lentement ; j'éprouve la résistance et j'entends la rumeur des distances traversées (Proust, R, I, p. 46, n. s.). 


\section{sous-vient en lui. Proust ne raconte pas ses souvenirs vécus, mais à travers eux, à chaque fois, immanquablement, inexorablement, avec le souvenir qui remonte, ce que ce souvenir traîne avec lui, soit la Nuit de la béance. Et c'est uniquement par là, par la nuit, qu'ils sont, ces souvenirs, « littéraires » : \\ L'auréole d'éternité qui nimbe le surgissement du passé, véritable résurrection, se détache et flotte dans sa fragilité sur le néant qu'elle repousse, un moment, distance précaire qui ouvre l'espace de l'œuvre. D'où la joie de la victoire sur la mort et l'oubli. C'est l'ensemble de ce mouvement, depuis l'abîme, qui est seul digne d'être narré et qui permet de ne plus douter de la valeur de la littérature ${ }^{13}$. Tel est ce qui donc fonde le devenir écrivain véritable, à la fois contemporain et antérieur, sous-jacent aux devenirs autres et multiples qui occupent sans doute trop bruyamment toute l'attention du nomadisme contemporain.}

La distance s'entend, et aussi l'abîme de nuit qui colle à ce qui remonte ou revient, et se

\section{Le corps sans organe du sujet de l'écriture}

Le devenir écrivain ne suppose ni moi ni sujet souverain. Il suppose un mode de subjectivation particulier pour lequel le sujet est d'abord une place vide (et pas du tout un sujet actif, synthétique). C'est un lieu de passage pour les différents devenirs. C'est en ce sens-là qu'il y a bien un sujet de l'écriture, engendré dans un devenir propre. C'est un être sans corps, un être entre la vie et la mort qui du fond de la destruction universelle et sa ligne d'abolition revoit la vie parcourue, l'histoire collective des peuples et des classes sociales pour leur dresser le monument d'éternité qu'est toute œuvre d'art. Cette demi-existence, faite d'ombre par sa proximité avec la nuit du temps et de l'oubli, est la seule a pouvoir passer par tous les devenirs. Sans cette ombre, pur corps sans organe imperceptible, qui se glisse sous eux, il n'y aurait ni devenir enfant, ni devenir femme, ni devenir-animal. Le devenir imperceptible, l'évanouissement dit moléculaire, est bien le sens des devenirs, puisqu'il en est le point de départ et la possibilité.

56 La subjectivité écrivante, qui n'est pas un sujet donné mais qui se produit dans et par l'écriture, n'est pas réduit à l'individu: elle le déborde ou dépasse, car elle est impersonnelle, l'Impersonnel même. Et en même temps, c'est un devenir-animal parce que cette subjectivation est en même temps l'apparition d'un sujet larvaire. C'est un devenir larve que le devenir écrivain.

Minorer l'homme, «s'arracher à l'humanité » (Mille plateaux, p. 294), sortir de la forme homme, devenir femme, enfant puis animal, imperceptible, tous ces devenirs n'ont pu être possible que parce que déjà un sujet sans moi, c'est-à-dire sans identification, sans attache ou, comme dit Deleuze, sans territorialisation, était en devenir devant le Temps, la mort, l'universel oubli, le non-sens... Pourquoi la musique fait-elle pleurer et donne-t-elle tant envie de mourir ? Non parce que serait à l'œuvre une pulsion de mort qui nous précipiterait hors de la vie, mais parce que l'entrée dans l'art ou l'écriture est l'entrée dans une vie non-organique, qui se fait en empruntant une ligne abstraite, qui, tel Orphée, nous conduit aux Enfers, soit aux limites extrêmes de notre condition. Toute grande œuvre est d'abord l'épreuve du temps, de l'inexorable abolition, de la béance, même celle qui paraît la plus insouciante, la plus innocente, comme celle qui 
par exemple s'intitule « À l'ombre des jeunes filles en fleur ». Mais, je laisse le dernier mot à Gilles Deleuze, qui concernant la nécessité de la rencontre de la béance dit à propose d'une œuvre comme celle de Watteau :

\section{NOTES}

L'art, écrit Gilles Deleuze, lutte avec le chaos, mais pour le rendre sensible, même à travers le personnage le plus charmant, le paysage le plus enchanté (Watteau) (QQPh, p. 192).

1. Cet ouvrage, édité chez Minuit, sera cité par l'abréviation «Kafka » en italique. Mille plateaux, est édité chez Minuit, en 1980 (= MP). Dialogue renvoie à Claire PARNET et Gilles DELEUZE, Flammarion, 1977. Le sigle Cr\&cl, renvoie à Critique et clinique, Éditions de Minuit, 1993, et QQPh, à Qu'est-ce que la philosophie ?, Éditions de Minuit, 1990. Le sigle R, suivi de chiffres romains, renvoie à La Recherche du temps perdu et aux trois tomes édités dans la Pléiade, Gallimard, en 1954.

2. . Le tiret qui accole Proust et Kafka, tend à signifier que c'est un Proust saisi à travers sa propre compréhension de Kafka qu'à partir de 1975 Deleuze lira Proust. Ce qui n'est pas le cas du premier essai de 1964, Proust et les signes. Il y a plusieurs strates dans la lecture de Proust par Deleuze. Les ignorer, c'est ouvrir la porte aux contresens. Voir sur ce problème, mon intervention au colloque de Cardiff le 10 Mars 2006, Proust, Deleuze, Beckett, « Mnémosyne : déesse ou usine ?». Et, Le Point littéraire, à paraître.

3. Voir aussi un passage comme «la matière de mon expérience, laquelle serait la matière de mon livre ... » R, III, 915 ; et R, III,1032 : la vie « en somme réalisée dans un livre » et le bonheur d'écrire un tel livre, etc.

4. . Voir sur l'opposition grotesque de la vie et de l'écriture, Kafka, p. 74, et Dialogue, p. 169 : il y a un seul et même processus; en même temps que la vie (dont s'occupe la clinique) cesse d'être personnelle, l'œuvre (dont s'occupe la critique) cesse d'être littéraire ou textuelle; elles sont une seule et même chose (critique et clinique, œuvre et vie) « quand elles ont épousé la ligne de fuite qui en fait les pièces d'une même machine de guerre ».

5. . Tout ce qui change, dit Deleuze, passe par cette ligne, Dialogue, p. 91. On peut prendre en exemple de vie non organique, « la vie qu'il peut y avoir dans une ligne de dessin, d'écriture ou de musique ", Pourparlers, p. 196.

6. . Ce qui différencie à vrai dire la ligne abstraite de la ligne de fuite, c'est que la première est nécessairement présente en tout agencement, alors que ce dernier peut fonctionner sans ligne de fuite ou en l'écrasant, la bouchant, voir Dialogue, p. 128-129. La ligne de fuite est une ligne de variation qui affecte un système, un ensemble, en l'empêchant d'être homogène (Dialogue, p. 11).

7. . Pourparlers, p. 144. Voir principalement Mille plateaux, p. 357-358; 363.

8. . Paul valéry, Cuvres, Pléiade, II, p. 629. Il disait aussi : « Le véritable ouvrier d'un bel ouvrage... n'est positivement personne » (Ibid., I, p. 483). Pour tout ceci, voir G. GENETTE, Figures, I, p. 259.

9. . Ibid., II, p. 581.

10. . Ibid. II, p. 575.

11. . Voir Mille plateaux, p. 360, les devenirs sont une « anti-mémoire».

12. . «Un énorme Corps sans organes ", dit Deleuze. Voir la folie du narrateur telle qu'elle est décrite dans l'édition de 75 de Proust et les signes, p. 217 et s.

13. . Voir, par exemple, Le Temps retrouvé, R, III, p. 814. 


\section{RÉSUMÉS}

Gilles Deleuze et Félix Guattari opèrent un tri entre les devenirs et le devenir-écrivain. Pourquoi une telle exclusion? Quelles sont ses conditions de possibilité ? Le cas de Proust semble faire objection, lui, dont la vie et l'œuvre sont le récit d'une telle vocation. Une analyse de l'œuvre de Deleuze qui garde fidèlement la ligne tracée dans la continuité de Logique du sens (1969) permet de dégager, tant chez Deleuze que chez Proust, un même sujet de l'écriture. Ce mode de subjectivation et ce devenir se définissent par la perte de l'Ego et la confrontation au non sens du «Chaos». Cette écheveau de lignes, qui est connecté au réel de la béance du sens, traverse continûment, mais discrètement, jusqu'aux derniers articles (reformulation en 1993, de la Préface à Wolfson, Le Schizo et les Langues), l'œuvre de Deleuze, et se maintient vivace sous le bruyant et tapageur discours du nomadisme et des déterritorialisations mondialistes.

Becomings, becoming writer, Proust-Kafka Gilles Deleuze and Felix Guattai are picking out the becoming-writer from the becomings in general. Why such a selection and exclusion? Proust's case seems to argue against such a sorting, whose «La Recherche » is a writer-vocation story. An examination of Deleuze's works, which is able to keep clearly the ligne of escape of Logique du sens (1969), give chance to a subject of writing, in Deleuze's works as well in Proust's works. This way of subjectivation and becoming are defined by the fading of the Ego and the confrontation to the void of sense, as Chaos. It is not a new interpretation of Deleuze, but a ligne of thought which is present along his works, until 1993 (in Preface to Wolfson, Le Schizo et les Langues), always working under the fashion and loud discourse of world-nomadism and global déterritorialisation.

Werdegänge, Schriftsteller werden, Proust-Kafka Eine Analyse des Werkes von Deleuze, die getreu die in der Kontinuität „der Logik des Sinnes“ gezogene Linie verfolgt, erlaubt es ein gleiches Subjekt des Schreibens, so bei Deleuze wie bei Proust, an den Tag treten zu lassen.

\section{AUTEUR}

\section{PHILIPPE MENGUE}

Philippe Mengue est Agrégé et Docteur d'État en philosophie. Il a enseigné à l'Université d'AixMarseille et au Collège International de Philosophie à Paris. Il a publié Gilles Deleuze ou le Système du multiple, Kimé, 1994, L'Ordre sadien, Kimé, 1996 ; Deleuze et la question de la démocratie, L'Harmatan, 2003 ; La Philosophie au piège de l'histoire, La Différence, 2004 et de nombreux articles dans différentes revues, françaises et anglaises, américaines et canadiennes. 